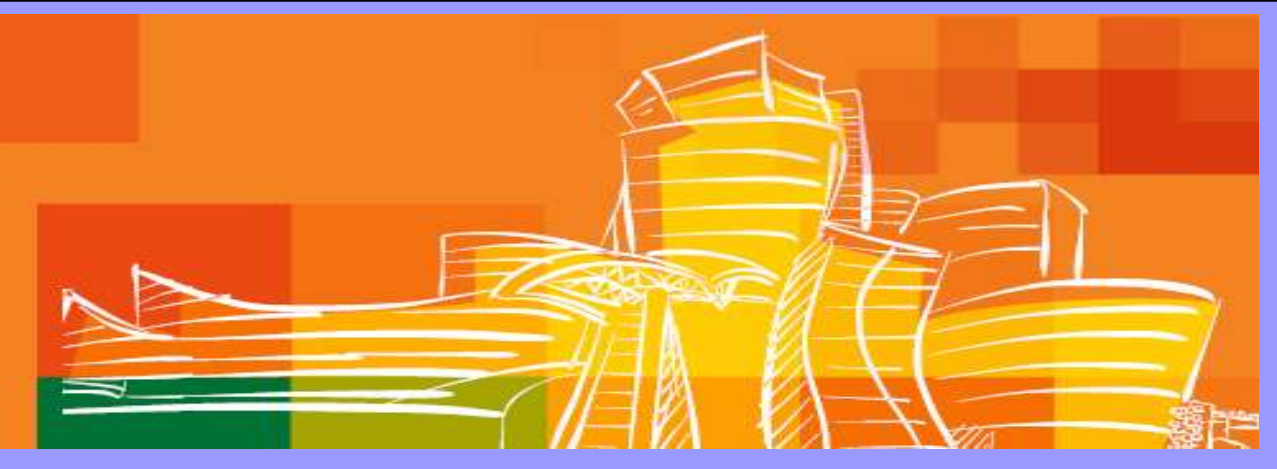

\title{
PECS II BLOCK AS AN EFFECTIVE OPTION FOR PRIMARY ANESTHESIA DURING BREAST SURGERY
}

Ospedale
Evangelico
Betania
fondazione evangelica betania

Background and Aims

PECS II block may be adopted as an effective anesthesiologic alternative for breast surgery when general anesthesia is contraindicated. We here report a successful case of PECS II block for the excision of breast's outer quadrants.

\section{Methods}

A 68-years-old woman, with a body mass index of 64.4 and several comorbidities [hypertension, diabetes, atrial fibrillation, obstructive sleep apnea syndrome and chronic obstructive pulmonary disease under chronic oxygentherapy], was affected by recurrent breast cancer requiring surgery. Anesthesiologic preoperative evaluation showed a difficult tracheal intubation index (el-Ganzouri 7), the patient was thus considered a candidate to PECS II BLOCK and signed an informed consent. In the operating room, she was placed in lateral decubitus position with her right arm flexed above head, under continuous $\mathrm{O}_{2}(2 \mathrm{l} / \mathrm{min})$ through nasal glasses and vital signs monitoring, Sufentanyl was administered i.v. (5 mcg) and ultrasound-guided PECS II block was performed. A high-frequency linear probe and a $22 \mathrm{G}$ hybrid needle evolution $($ TEMENA®) were used. Ropivacaine $(0,75 \%$, $20 \mathrm{ml}$ ) was injected between serratus anterior and pectoralis minor muscles at the intersection of fourth rib and anterior axillary line. Sedation with Propofol (1-2 mg/kg/h) was performed. EtCO was monitored.

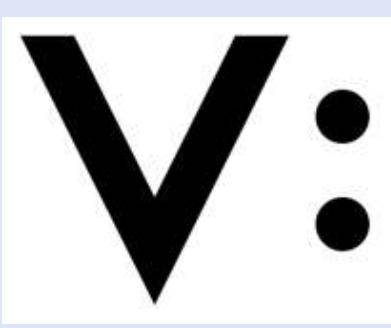

Università

degli Studi

della Campania

Luigi Vanvitelli
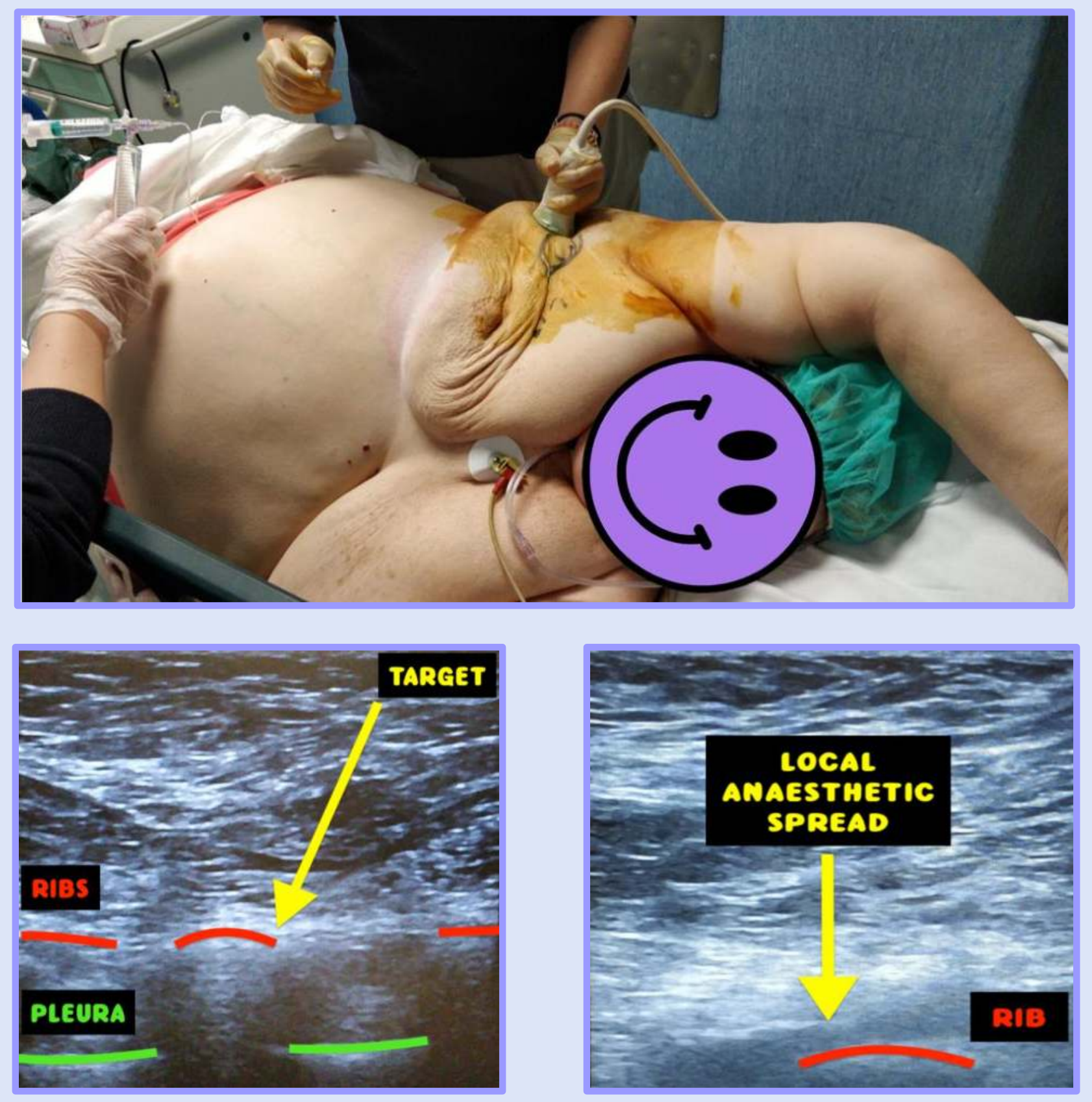

Results

Surgical procedure was performed 45 minutes after PECS II block. The patient remained awake during the procedure, in spontaneous breathing. Vital signs remained stable and normal until the end of procedure.

\section{Conclusions}

PECS II block may represent an effective option in patients affected by several comorbidities contraindicating general anesthesia, due to its simplicity and a relatively low incidence of side effects. 